\title{
Variational and Conformal Structure of Nonlinear Metric-connection Gravitational Lagrangians
}

\author{
Spiros Cotsakis $a$ \\ Department of Mathematics, University of the Aegean, Karlovassi 83200, Samos, Greece \\ John Miritzis $b$ \\ Department of Mathematics, University of the Aegean, Karlovassi 83200, Samos, Greece \\ Laurent Querellac) \\ Institut d'Astrophysique et de Géophysique, Université de Liège, Avenue de Cointe 5, B-4000 Liège, Belgium
}

(February 7, 2008)

\begin{abstract}
We examine the variational and conformal structures of higher order theories of gravity which are derived from a metric-connection Lagrangian that is an arbitrary function of the curvature invariants. We show that the constrained first order formalism when applied to these theories may lead consistently to a new method of reduction of order of the associated field equations. We show that the similarity of the field equations which are derived from appropriate actions via this formalism to those produced by Hilbert varying purely metric Lagrangians is not merely formal but is implied by the diffeomorphism covariant property of the associated Lagrangians. We prove that the conformal equivalence theorem of these theories with general relativity plus a scalar field, holds in the extended framework of Weyl geometry with the same forms of field and self-interacting potential but, in addition, there is a new 'source term' which plays the role of a stress. We point out how these results may be further exploited and address a number of new issues that arise from this analysis.
\end{abstract}

04.20.Fy, 04.50.+h

\section{INTRODUCTION}

According to the standard variational principle (Hilbert variation) that leads to the Einstein field equations of general relativity, the gravitational action $\int R \sqrt{-g}$ is varied with respect to the metric tensor of a spacetime manifold that is taken to be a four-dimensional Lorentz manifold $(\mathcal{M}, \mathbf{g})$ with metric $\mathbf{g}$ and the Levi-Civita connection $\nabla$. However, in many instances (see Ref. 1 for a complete review) one considers a Lorentz manifold with an arbitrary connection $\not \nabla$ that is incompatible with the metric, i.e. $\nabla \mathbf{g} \neq 0$. A motivation for such a generalization was initially inspired by early work of Weyl (Ref. 2). In this case, one considers variation of an appropriate action with respect to both the metric components $g_{a b}$ and the connection coefficients $\Gamma_{b c}^{a}$ without imposing from the beginning that $\Gamma_{b c}^{a}$ be the usual Christoffel symbols. In the current literature this variational principle, where the metric and the connection are considered as independent variables, is referred to as the first order or metric-connection or simply Palatini variation. (For an historical commentary of this principle of variation and related issues, we refer to Ref. 3.)

Such alternative variational methods were first analysed in the framework of nonlinear gravitational Lagrangians by Weyl (Ref. 2), Eddington (Ref. 4) and others (Ref. 5 - 20). In an effort to obtain second order field equations different from Einstein's, Stephenson (Ref. 5.,6) and Higgs (Ref. (7) applied the first order formalism to the quadratic Lagrangians $R^{2}, R_{a b} R^{a b}, R_{a b c d} R^{a b c d}$ and Yang (Ref. E) investigated the Lagrangian $R_{a b c d} R^{a b c d}$, by analogy with the Yang-Mills Lagrangian. However, Buchdahl (Ref. 9) pointed out a difficulty associated with this version of the metric-connection variation which is related to imposing the metricity condition, i.e. the connection coefficients equal to the Christoffel symbols, after completing the variation, and subsequently constructed specific examples showing that this version of the first order formalism is not a reliable method in general (Ref. 10). Van den Bergh (Ref. 11) arrived at a similar conclusion in the context of general scalar-tensor theories. The $R+\alpha R^{2}$ theory including matter was investigated in this framework by Shahid-Saless (Ref. 12) and generalized to the $f(R)$ case by Hamity and Barraco (Ref. 13). These authors also studied conservation laws and the weak field limit of the resulting equations. More recently, Ferraris et al. (Ref. 14) showed that the first order formalism applied to general $f(R)$ vacuum Lagrangians leads to a series of Einstein spaces with cosmological constants determined by the explicit form of the function $f$. Similar results were obtained in the case of $f\left(\right.$ Ric $\left.^{2}\right)$ theories by Borowiec et al. (Ref. 15).

A consistent way to consider independent variations of the metric and connection in the context of Riemannian geometry is to add a compatibility condition between the metric and the connection as a constraint with Lagrange 
multipliers. In vacuum general relativity, this constrained first order formalism results in the Lagrange multipliers vanishing identically as a consequence of the field equations (Ref. 16). This method was applied to quadratic Lagrangians with the aim of developing a Hamiltonian formulation for these theories in Ref. 17.

Consider a Lorentzian manifold $(\mathcal{M}, \mathbf{g}, \nabla)$ of dimension $\mathrm{D}$ where $\not \nabla$ is an arbitrary symmetric connection. Hence, $\not \nabla \mathbf{g} \neq 0$ that is, the connection coefficients are functions independent of the metric components and the Ricci tensor is a function of the connection only. In the case of general relativity without matter fields, varying the corresponding action

$$
S=\int L \sqrt{-g} \mathrm{~d}^{\mathrm{D}} x, \quad L=g^{m n} R_{m n}
$$

one arrives at the well-known result that variation with respect to the metric produces vacuum Einstein's equations whereas variation with respect to the connection reveals that the connection is necessarily the Levi-Civita connection (provided that $\mathrm{D} \neq 2$ ). The integral (1) is taken over a compact region $\mathcal{U}$ of the spacetime $(\mathcal{M}, \mathbf{g}, \mathbb{X})$ and we assume that the metric and the connection are held constant on the boundary of $\mathcal{U}$. In the sequel we omit the symbol $\mathrm{d}^{\mathrm{D}} x$ under the integral sign and set $w:=\sqrt{-g}$. Gothic characters denote tensor densities, for example $\mathfrak{g}_{a b}:=w g_{a b}$.

In the presence of matter fields there is an ambiguity because the compatibility condition between the metric and the connection does not hold. The matter Lagrangian depends primarily on the field variables $\psi$, and assumes a form that is a generalization of its special relativistic form, which is achieved via the strong principle of equivalence and the principle of minimal coupling, according to the scheme $\eta_{a b} \rightarrow g_{a b}$ and $\partial \rightarrow \not \nabla$, (the order of the two steps being irrelevant as long as the connection is the Levi-Civita one). Variation of the total action

$$
S=\int\left[\mathfrak{R}(g, \Gamma)+\mathfrak{L}_{\mathrm{m}}(g, \psi, \not \nabla \psi)\right],
$$

gives the following pair of equations

$$
\begin{gathered}
G_{a b}=T_{a b}:=-\frac{2}{w} \frac{\delta \mathfrak{L}_{\mathrm{m}}}{\delta g^{a b}}, \\
\delta_{c}^{b} \not_{d} \mathfrak{g}^{a d}+\delta_{c}^{a} \not_{d} \mathfrak{g}^{b d}-2 \not_{c} \mathfrak{g}^{a b}=2 \frac{\delta \mathfrak{L}_{\mathrm{m}}}{\delta \Gamma_{a b}^{c}} .
\end{gathered}
$$

These equations are inconsistent in general unless the matter Lagrangian does not depend explicitly on the connection, i.e. $\delta \mathfrak{L}_{\mathrm{m}} / \delta \Gamma_{a b}^{c}=0$. (It is interesting to note that in the case $\mathrm{D}=2$ in vacuum, since the equation $\Gamma_{a b}^{c}=\left\{\begin{array}{l}c \\ a b\end{array}\right\}+1 / 2\left(\delta_{a}^{c} Q_{b}+\delta_{b}^{c} Q_{a}-g_{a b} Q^{c}\right)$, with $Q_{a}:=-\nabla_{a} \ln w=-\partial_{a} \ln w+\Gamma_{a}$, and $\Gamma_{a}:=\Gamma_{a b}^{b}$, has a vanishing trace, $(1-\mathrm{D} / 2)\left(\partial_{a} \ln \sqrt{-g}-\Gamma_{a}\right)=0$, the $\Gamma_{a}$ part of the connection is undetermined (Ref. 18).)

In Section 【1 theories, we show how the constrained first order formalism is used to prove that the field equations of these theories can be given in a reduced form that makes the comparison to the usual Hilbert equations direct and point out that such a correspondence is due to the diffeomorphism covariance property of the associated Lagrangians. In Section III, we prove that in Weyl geometry, higher order gravity theories are conformally equivalent to general relativity plus a scalar field matter source with the same self-interacting potential as in the standard Riemannian case, together with a new 'source term' that arises as a result of the presence of the Weyl covariant vectorfield. In the last section, we comment on the usefulness of the extended form of the conformal equivalence and point out how these results may be further exploited to develop this framework.

\section{CONSTRAINED AND UNCONSTRAINED VARIATIONS}

We begin with a Lagrangian that is a smooth function of the scalar curvature $R$ and vary the corresponding action

$$
S=\int w f(R)
$$

with respect to the metric tensor and the connection to obtain respectively

$$
f^{\prime} R_{(a b)}-\frac{1}{2} f g_{a b}=0
$$




$$
\nabla_{a}\left(w f^{\prime} g^{b c}\right)=0
$$

Explicitly, the $\Gamma$-equation (5b) reads

$$
\left(\partial_{a} \ln w+\left(\ln f^{\prime}\right)^{\prime} \partial_{a} R-\Gamma_{a}\right) g_{b c}-\partial_{a} g_{b c}+\Gamma_{b a}^{m} g_{m c}+\Gamma_{c a}^{m} g_{m b}=0,
$$

and so we can solve for $\Gamma_{a}$ and substitute back in (6) to find

$$
\partial_{a} \widetilde{g}_{b c}=\Gamma_{b a}^{m} \widetilde{g}_{m c}+\Gamma_{c a}^{m} \widetilde{g}_{m b},
$$

where we have introduced a new metric $\widetilde{g}_{a b}:=f^{\prime} g_{a b}$, with conformal factor $f^{\prime}$. This means that $\Gamma$ is the Levi-Civita connection for the metric $\widetilde{\mathrm{g}}$.

Equation (5a) is more straightforward. On the one hand, its trace $f^{\prime}(R) R=2 f(R)$ is satisfied identically if $f(R)=\alpha R^{2}$, (up to a constant rescaling factor $\alpha$ ) and so (5a) becomes $R_{a b}-(1 / 4) R g_{a b}=0$, (provided that $\left.f^{\prime}(R) \neq 0\right)$ which finally gives $\widetilde{R}_{a b}-(1 / 2 \alpha) \widetilde{g}_{a b}=0$ so that the underlined manifold is an Einstein space with constant scalar curvature $\widetilde{R}=\widetilde{g}^{a b} \widetilde{R}_{a b}=2 / \alpha$. On the other hand, one could regard the above trace as an algebraic equation in $R$ with roots $\rho_{1}, \rho_{2}, \ldots$. This situation was analysed by Ferraris et al. (Ref. 14) who showed that such an analysis leads to a series of Einstein spaces each having a constant scalar curvature (see also Ref. 10).

By a completely analogous procedure one finds Einstein spaces for the choice $L=f(r)$ where $r=Q_{a b} Q^{a b}$, and $Q_{a b}$ is the symmetric part of the Ricci tensor and also for the Lagrangian $L=f(K)$ where $K=R_{a b c d} R^{a b c d}$. Note that in this last case, varying the corresponding action with respect to the metric and the connection, one obtains

$$
-\frac{1}{2} f g_{a b}-f^{\prime} R_{a}{ }^{k l m} R_{b k l m}+f^{\prime} R^{k}{ }_{a l m} R_{k b}{ }^{l m}+2 f^{\prime} R^{k}{ }_{l a m} R_{k b}{ }^{l m}=0
$$

and

$$
\nabla_{d}\left(w f^{\prime} R_{a}^{(b c) d}\right)=0
$$

with trace $f^{\prime}(K) K=f(K)$. Hence, either $f(K)=\alpha K$ identically or, given a function $f$, the trace is solved algebraically for $K$. In contrast to the previous cases, there exists no natural way to derive a metric $\widetilde{\mathbf{g}}$ from the field equation (9) unless the Weyl tensor vanishes (Ref. 19). Notice that the field equations derived from the Lagrangian $R_{[a b]} R^{a b}$ by the Palatini variation impose only four conditions upon the forty connection coefficients and leave the metric components entirely undetermined (Ref. 10).

Let us now introduce a vectorfield $Q_{c}$ called the Weyl covariant vectorfield and assume a linear metric-connection relation

$$
\nabla_{c} g_{a b}=-Q_{c} g_{a b}
$$

and define

$$
C_{a b}^{c}=\Gamma_{a b}^{c}-\left\{\begin{array}{l}
c \\
a b
\end{array}\right\}
$$

where,

$$
\Gamma_{a b}^{c}=\left\{\begin{array}{l}
c \\
a b
\end{array}\right\}+\frac{1}{2} g^{c m}\left(Q_{b} g_{a m}+Q_{a} g_{m b}-Q_{m} g_{a b}\right) .
$$

The constrained first order formalism consists of adding to the original Lagrangian the following term as a constraint (with Lagrange multipliers $\Lambda$ )

$$
L_{\mathrm{c}}(g, \Gamma, \Lambda)=\Lambda_{r}^{m n}\left[\Gamma_{m n}^{r}-\left\{\begin{array}{l}
r \\
m n
\end{array}\right\}-C_{m n}^{r}\right] .
$$

For instance, in Riemannian geometry (13) takes the form

$$
L_{\mathrm{c}}(g, \Gamma, \Lambda)=\Lambda_{r}^{m n}\left[\Gamma_{m n}^{r}-\left\{\begin{array}{c}
r \\
m n
\end{array}\right\}\right],
$$

while, in Weyl geometry

$$
L_{\mathrm{c}}(g, \Gamma, \Lambda)=\Lambda_{r}^{m n}\left[\Gamma_{m n}^{r}-\left\{\begin{array}{l}
r \\
m n
\end{array}\right\}-\frac{1}{2} g^{r s}\left(Q_{n} g_{m s}+Q_{m} g_{s n}-Q_{s} g_{m n}\right)\right]
$$


As an example, consider any of the previous test Lagrangians $L(g, \Gamma, \psi)$ and vary the resulting action

$$
S=\int w\left[L(g, \Gamma, \psi)+L_{\mathrm{c}}(g, \Gamma, \Lambda)\right]
$$

with respect to the independent fields $g, \Gamma, \Lambda$ and $\psi$. We find the $g$-equations

$$
\left.\frac{\delta(w L)}{\delta g^{a b}}\right|_{\Gamma}+w B_{a b}=0
$$

where $B_{a b}$ is defined by

$$
B_{a b}:=-\frac{1}{2} \not \nabla^{m}\left[\Lambda_{b a m}+\Lambda_{a m b}-\Lambda_{m a b}\right]
$$

and the $\Gamma$-equations

$$
\left.\frac{\delta L}{\delta \Gamma_{a b}^{c}}\right|_{g}+\Lambda_{c}^{a b}=0
$$

Variation with respect to the matter fields $\psi$ yields their respective equations of motion.

In the Riemannian case in particular we have (some of the expressions given below appear incorrectly in Ref. 17), for the Lagrangian $L=R^{2}$,

$$
\begin{gathered}
\frac{1}{2} R^{2} g^{a b}-2 R R^{a b}+B^{a b}=0 \\
\Lambda_{c}^{a b}=\left(2 g^{a b} \delta_{c}^{m}-g^{a m} \delta_{c}^{b}-g^{m b} \delta_{c}^{a}\right) \not \nabla_{m} R \\
B^{a b}=-2 g^{a b} \not \nabla R+2 \not \nabla^{b} \nabla^{a} R,
\end{gathered}
$$

for the Lagrangian $L=R_{m n} R^{m n}$,

$$
\begin{gathered}
\frac{1}{2} R_{m n} R^{m n} g^{a b}-R^{a m} R_{n}^{b}-R_{m}{ }^{b} R^{m a}+B^{a b}=0 \\
\Lambda_{c}^{a b}=2 \not_{c} R^{a b}-\delta_{c}^{a} \nabla_{m} R^{m b}-\delta_{c}^{b} \nabla_{m} R^{a m} \\
B^{a b}=-\not \triangleright R^{a b}+2 \nabla_{m} \not^{b} R^{a m}-g^{a b} \nabla_{n} \nabla_{m} R^{m n},
\end{gathered}
$$

for the Lagrangian $L=R_{m n r s} R^{m n r s}$,

$$
\begin{gathered}
\frac{1}{2} R_{m n r s} R^{m n r s} g^{a b}-2 R^{a m n r} R_{m n r}^{b}+B^{a b}=0 \\
\Lambda_{a}^{b c}=2 \not_{m} R_{a}^{b c m}+2 \nabla_{m} R_{a}^{c b m} \\
B^{a b}=4 \not_{n} \nabla_{m} R^{a m b n}
\end{gathered}
$$

and for the Lagrangian $L=f(R)$,

$$
\begin{gathered}
\frac{1}{2} f g_{a b}-f^{\prime} R_{(a b)}+B_{a b}=0 \\
\left(2 g^{b c} \delta_{a}^{m}-g^{m c} \delta_{a}^{b}-g^{b m} \delta_{a}^{b}\right) \nabla_{m} f^{\prime}=\Lambda_{a}^{b c}
\end{gathered}
$$




$$
B^{a b}=-g^{a b} \not f^{\prime}+\not \nabla^{b} \not \nabla^{a} f^{\prime}
$$

It is straightforward to obtain the correspondence with the Hilbert case by substituting the $B_{a b}$ 's in the first equation in each of these above cases. They read respectively

$$
\begin{gathered}
\frac{1}{4} R^{2} g^{a b}-R R^{a b}+\nabla^{b} \nabla^{a} R-g^{a b} \square R=0 \\
\frac{1}{2} R_{m n} R^{m n} g^{a b}-2 R^{b m a n} R_{m n}+\nabla^{b} \nabla^{a} R-\square R^{a b}-\frac{1}{2} \square R g^{a b}=0 \\
\frac{1}{2} R_{m n r s} R^{m n r s} g^{a b}-2 R^{m n r b} R_{m n r}^{a}+4 \nabla_{n} \nabla_{m} R^{a m n b}=0 \\
f^{\prime} R_{(a b)}-\frac{1}{2} f g_{a b}-\nabla_{a} \nabla_{b} f^{\prime}+g_{a b} \square f^{\prime}=0 .
\end{gathered}
$$

Strictly speaking, compared to the usual Hilbert variation, in all cases considered so far using the first order formalism, one starts from a different lagrangian defined in a different function space, follows a different method and ends up in a different set of field equations. Bearing this in mind, one nevertheless sees the striking resemblance of the expressions obtained via the two variational procedures (Equations (18 22)). We shall now see this is not a mere formal coincidence but that the reason lies in the fact that all our test Lagrangians are diffeomorphism covariant.

All previous cases can be considered as specializations derived from a very general Lagrangian $n$-form constructed locally as follows,

$$
L=L\left(g_{a b}, \nabla_{a_{1}} g_{a b}, \ldots, \nabla_{\left(a_{1} \ldots\right.} \nabla_{\left.a_{k}\right)} g_{a b}, \psi, \nabla_{a_{1}} \psi, \ldots, \nabla_{\left(a_{1} \ldots\right.} \nabla_{\left.a_{l}\right)} \psi, \gamma\right)
$$

that is, $L$ is a function of dynamical fields $g, \psi$ and also of other fields collectively referred to as $\gamma$. Referring to $g$ and $\psi$ as $\phi, L$ is called $f$-covariant, $f \in \operatorname{Diff}(M)$, or simply diffeomorphism covariant if,

$$
L\left(f^{*}(\phi)\right)=f^{*} L(\phi),
$$

where $f^{*}$ denotes the induced action of $f$ on the fields $\phi$. Note that this definition excludes the action of $f^{*}$ on $\not \nabla$ or the other fields $\gamma$. It immediately follows that our test Lagrangians considered previously satisfy the above definition and as a result are diffeomorphism covariant.

It is a very interesting result, first shown by Iyer and Wald (Ref. 21) that if $L$ in (23) is diffeomorphism covariant, then $L$ can be re-expressed in the form

$$
L=L\left(g_{a b}, R_{b c d e}, \nabla_{a_{1}} R_{b c d e}, \ldots, \nabla_{\left(a_{1} \ldots\right.} \nabla_{\left.a_{m}\right)} R_{b c d e}, \psi, \nabla_{a_{1}} \psi, \ldots, \nabla_{\left(a_{1} \ldots\right.} \nabla_{\left.a_{l}\right)} \psi\right),
$$

where $\nabla$ denotes the Levi-Civita connection of $g_{a b}, R_{a b c d}$ denotes the Riemann curvature of $g_{a b}$ and $m=\max (k-$ $2, l-2)$. Notice that everything is expressed in terms of the Levi-Civita connection of the metric tensor and also that all other fields $\gamma$ are absent.

Applying the Iyer-Wald theorem in our test Lagrangians we immediately see that we could have re-expressed them from the beginning in a form that involves only the Levi-Civita connection and not the original arbitrary connection $\not \nabla$, and vary them to obtain the corresponding 'Hilbert' equations. As we showed above, we arrived at this result by treating the associated Lagrangians as different (indeed they are!) according to whether or not they involved an arbitrary (symmetric) or a Levi-Civita connection.

\section{CONFORMAL STRUCTURE AND WEYL GEOMETRY}

For the more general nonlinear Lagrangians of the form $f(q)$ where $q=R, R_{a b} R^{a b}$, or $R_{a b c d} R^{a b c d}$ where $f$ is an arbitrary smooth function considered in the previous section, the field equations obtained by the metric-connection formalism are of second order while the corresponding ones obtained via the usual metric variation are of fourth order. This result sounds very interesting since it could perhaps lead to an alternative way to 'cast' the field equations of these theories in a more tractable, reduced form than the one that is usually used for this purpose namely, the 
conformal equivalence theorem (Ref. 22). In this way, certain interpretational issues related to the question of the physicality of the two metrics (Ref. 23,24) associated with the conformal transformation would perhaps be avoided.

As discussed in the previous section, the constraint (13) for Weyl geometry becomes

$$
L_{\mathrm{c}}(g, \Gamma, \Lambda)=\Lambda_{c}^{a b}\left[\Gamma_{a b}^{c}-\left\{\begin{array}{c}
c \\
a b
\end{array}\right\}-\frac{1}{2} g^{c m}\left(Q_{a} g_{m b}+Q_{b} g_{a m}-Q_{m} g_{a b}\right)\right] .
$$

In order to examine the consequences of the Weyl constraint (26) we now apply the constrained first order formalism to the Lagrangian $L=f(R)$. Variation with respect to the Lagrange multipliers recovers the expression (12) of the Weyl connection. Variation with respect to the metric yields the $g$-equations

$$
f^{\prime} R_{(a b)}-\frac{1}{2} f g_{a b}+B_{a b}=0
$$

where $B_{a b}$ is defined by (16). Variation with respect to the connection yields the explicit form of the Lagrange multipliers, namely,

$$
\Lambda_{c}^{a b}=\frac{1}{2} \delta_{c}^{b}\left(Q^{a} f^{\prime}-\not \nabla^{a} f^{\prime}\right)+\frac{1}{2} \delta_{c}^{a}\left(Q^{b} f^{\prime}-\not \nabla^{b} f^{\prime}\right)-g^{a b}\left(Q_{c} f^{\prime}-\not \nabla_{c} f^{\prime}\right) .
$$

Substituting back this last result into equation (16) we find,

$$
B_{a b}=2 Q_{(a} \nabla_{b)} f^{\prime}-\nabla_{(a} \nabla_{b)} f^{\prime}+f^{\prime} \nabla_{(a} Q_{b)}-f^{\prime} Q_{a} Q_{b}-g_{a b}\left(2 Q_{m} \not \nabla^{m} f^{\prime}-Q^{2} f^{\prime}-\not \supset f^{\prime}+f^{\prime} \not \nabla^{m} Q_{m}\right) .
$$

Inserting this result into equation (27) we obtain the full field equations for the Lagrangian $L=f(R)$ in the framework of Weyl geometry, namely,

$$
f^{\prime} R_{(a b)}-\frac{1}{2} f g_{a b}-\not \nabla_{a} \not \nabla_{b} f^{\prime}+g_{a b} \not \triangleright f^{\prime}=M_{a b},
$$

where $M_{a b}$ is defined by

$$
M_{a b}=-2 Q_{(a} \nabla_{b)} f^{\prime}-f^{\prime} \nabla_{(a} Q_{b)}+f^{\prime} Q_{a} Q_{b}+g_{a b}\left(2 Q_{m} \not \nabla^{m} f^{\prime}-Q^{2} f^{\prime}+f^{\prime} \nabla^{m} Q_{m}\right) .
$$

It is interesting to note that the degenerate case $Q_{a}=0$ corresponds to the usual field equations obtained by the Hilbert variation in the framework of Riemann geometry, namely,

$$
f^{\prime} R_{a b}-\frac{1}{2} f g_{a b}-\nabla_{a} \nabla_{b} f^{\prime}+g_{a b} \square f^{\prime}=0 .
$$

It is known that these equations are conformally equivalent to Einstein equations with a self-interacting scalar field as the matter source (Ref. 22). In what follows, we generalize this property of the $f(R)$ field equations in Weyl geometry. To this end, we define the metric $\widetilde{g}$ conformally related to $g$ with $f^{\prime}$ as the conformal factor. Under a conformal transformation, the Weyl vectorfield transforms as

$$
\widetilde{Q}_{a}=Q_{a}-\not \nabla_{a} \ln f^{\prime}
$$

and the field equations (30) in the conformal frame read,

$$
f^{\prime} \widetilde{R}_{(a b)}-\frac{1}{2} \frac{f}{f^{\prime}} \widetilde{g}_{a b}-\widetilde{\nabla}_{a} \widetilde{\nabla}_{b} f^{\prime}+\widetilde{g}_{a b} \widetilde{\not} f^{\prime}=\widetilde{M}_{a b},
$$

where $\widetilde{\nabla}=\not \nabla, \widetilde{\not}=\widetilde{g}^{a b} \widetilde{\nabla}_{a} \widetilde{\nabla}_{b}=\left(f^{\prime}\right)^{-1} \not \triangleright$ and $\widetilde{M}_{a b}$ is given by

$$
\widetilde{M}_{a b}=f^{\prime} \widetilde{Q}_{a} \widetilde{Q}_{b}-f^{\prime} \widetilde{\nabla}_{(a} \widetilde{Q}_{b)}-\widetilde{\nabla}_{a} \widetilde{\nabla}_{b} f^{\prime}+\widetilde{g}_{a b}\left(f^{\prime} \widetilde{\nabla}^{m} \widetilde{Q}_{m}-f^{\prime} \widetilde{Q}^{2}+\widetilde{\not \nabla} f^{\prime}\right) .
$$

Introducing the scalar field $\varphi=\ln f^{\prime}$ and the potential $V(\varphi)$ in the 'usual' form (Ref. 22)

$$
V(\varphi)=\frac{1}{2}\left(f^{\prime}(R)\right)^{-2}\left(R f^{\prime}(R)-f(R)\right),
$$


we find that the field equations take the final form,

$$
\widetilde{G}_{a b}=\widetilde{M}_{a b}^{Q}-\widetilde{g}_{a b} V(\varphi),
$$

where,

$$
\widetilde{G}_{a b}=\widetilde{R}_{(a b)}-\frac{1}{2} \widetilde{R} \widetilde{g}_{a b}
$$

and

$$
\widetilde{M}_{a b}^{Q}=\widetilde{Q}_{a} \widetilde{Q}_{b}-\widetilde{\nabla}_{(a} \widetilde{Q}_{b)}+\widetilde{g}_{a b}\left(\widetilde{\nabla}^{m} \widetilde{Q}_{m}-\widetilde{Q}^{2}\right) .
$$

The field equations (34) could also be obtained from the corresponding conformally transformed Lagrangian using the constrained formalism. In that case, the equation of motion of the scalar field $\varphi$ derived upon variation with respect to $\varphi$ implies that the potential $V(\varphi)$ is constant.

Formally, the field equations (34) look like Einstein equations with a cosmological constant and a source term $\widetilde{M}_{a b}^{Q}$ depending on the field $\widetilde{Q}_{a}$. However, they cannot be identified as such unless the geometry is Riemannian, i.e. $\widetilde{Q}_{a}=0$. This will be the case only if the original Weyl vectorfield is a gradient, $Q_{a}=\nabla_{a} \Phi$. Then it can be gauged away by the conformal transformation $\widetilde{g}_{a b}=(\exp \Phi) g_{a b}$ and therefore the original space is not a general Weyl space but a Riemann space with an undetermined gauge (Ref. 25). This was the case of the unconstrained method applied to the Lagrangian $L=f(R)$, wherein the Weyl vector was deduced using equation (6) and turned out to be $Q_{a}=\nabla_{a}\left(\ln f^{\prime}\right)$. (In Ref. 20, this peculiarity is used in order to find out a subclass of theories based on a general D-dimensional dilaton gravity action, for which both unconstrained method and Hilbert variation yield dynamically equivalent systems.) This fact shows that unconstrained variations cannot deal with a general Weyl geometry and correspond to a degenerate case of the constrained method - the field equations obtained from the former can be recovered only by choosing specific forms of the Weyl vectorfield (Ref. 26).

\section{DISCUSSION}

The results obtained in Section II and Section III have the interpretation that a consistent way to investigate generalized theories of gravity without imposing from the beginning that the geometry is Riemannian, is the constrained first order formalism. Applications to quadratic and $f(R)$ Lagrangians in the framework of Riemannian and Weyl geometry reveal that unconstrained variational methods are degenerate cases corresponding to a particular gauge and that the usual conformal structure can be recovered in the limit of vanishing Weyl vector.

The generalization of the result stated above to include arbitrary connections with torsion can be an interesting exercise. The physical interpretation of the source term (equations (30) and (31)) is closely related to the choice of the Weyl vectorfield $Q$. However, it cannot be interpreted as a genuine stress-energy tensor in general since, for instance, choosing $Q$ to be a unit timelike, hypersurface-orthogonal vectorfield, the sign of $M_{a b} Q^{a} Q^{b}$ depends on the signs of $f^{\prime}(R)$ and the 'expansion' $\nabla_{a} Q^{a}$.

The generalization of the conformal equivalence theorem presented in Section III opens the way to analysing cosmology in the framework of these Weyl $f(R)$ theories by methods such as those used in the traditional Riemannian case. The first steps in such a program may be as follows (Ref. 27):

(A) Analyse the structure and properties of Friedmann cosmologies, find their singularity structure and examine the possibility of inflation.

(B) Consider the past and future asymptotic states of Bianchi cosmologies. Examine isotropization and recollapse conjectures in such universes. Look for chaotic behaviour in the Bianchi VIII and IX spacetimes.

(C) Formulate and prove singularity theorems in this framework. This will differ from the analysis in the Riemannian case (cf. Ref. 22) because of the presence of the source term $M_{a b}$.

All the problems discussed above can be tackled by leaving the conformal Weyl vectorfield $\widetilde{Q}_{a}$ undetermined while setting it to zero at the end will lead to detailed comparisons with the results already known in the Riemannian case.

\section{ACKNOWLEDGMENTS}

The work of S.C. and J.M. was supported by grants 3516075-2 and 1361/4.1 received from the General Secretariat of Science and Technology and the Research Commission of the University of the Aegean respectively, which are 
gratefully acknowledged. J.M. is grateful to the 'Institut d'Astrophysique et de Géophysique de l'Université de Liège' for kind hospitality while part of this work was done. L.Q. received support from a F.R.I.A. scholarship, from F.N.R.S. and A.R.C. grants. L.Q. wishes to acknowledge the kind hospitality of the Department of Mathematics of the University of the Aegean where part of this work was done.

a) E-mail: skot@math.aegean.gr

b) E-mail: imyr@kerkis.math.aegean.gr

c) E-mail: querella@astro.ulg.ac.be

${ }^{1}$ F. W. Hehl, J. D. McCrea, E. W. Mielke, and Y. Ne'eman, Phys. Rep. 258, 1 (1995).

${ }^{2}$ H. Weyl, Space-Time-Matter (Dover, New York, 1952).

${ }^{3}$ M. Ferraris, M. Francaviglia, and C. Reina, Gen. Rel. Grav. 14, 243 (1982).

${ }^{4}$ A. S. Eddington, The Mathematical Theory of Relativity (Cambridge U.P., Cambridge, 1922).

${ }^{5}$ G. Stephenson, Nuovo Cimento 9, 1959 (1958).

${ }^{6}$ G. Stephenson, Nuovo Cimento 9, 2085 (1958).

${ }^{7}$ P. Higgs, Nuovo Cimento 11, 816 (1958).

${ }^{8}$ C. N. Yang, Phys. Rev. Lett. 33, 445 (1974).

${ }^{9}$ H. Buchdahl, Proc. Camb. Phil. Soc. 56, 396 (1960).

${ }^{10}$ H. Buchdahl, J. Phys. A: Math. Gen. 12, 1229 (1979).

${ }^{11}$ N. Van den Bergh, J. Math. Phys. 22, 2245 (1981).

12 B. Shahid-Saless, Phys. Rev. D 35, 467 (1987).

${ }^{13}$ V. Hamity, and D. Barraco, Gen. Rel. Grav. 25, 461 (1993).

${ }^{14}$ M. Ferraris, M. Francaviglia, and I. Volovich, Class. Quantum Grav. 11, 1505 (1994).

${ }^{15}$ A. Borowiec, M. Ferraris, M. Francaviglia, and I. Volovich, Class. Quantum Grav. 15, 43 (1998).

${ }^{16}$ J. R. Ray, Nuovo Cimento 25B, 706 (1975).

17 J. Safko, and F. Elston, J. Math. Phys. 17, 1531 (1976).

18 S. Deser, Inequivalence of First and Second Order Formulations in D = 2 Gravity Models, gr-qc/9512022, 1995. See also references therein.

19 S. Davis, Gen. Rel. Grav. 30, 345 (1998).

${ }^{20}$ H. Burton, and R. B. Mann, Class. Quantum Grav. 15, 1375 (1998).

${ }^{21}$ V. Iyer, and R. M. Wald, Phys. Rev. D 50, 846 (1994). (cf. Lemma 2.1).

22 J. Barrow, and S. Cotsakis, Phys. Lett. B 214, 515 (1988).

${ }^{23}$ S. Cotsakis, Phys. Rev. D 47, 1437 (1993). Erratum, Phys. Rev. D 49, 1145 (1994).

${ }^{24}$ S. Cotsakis, Phys. Rev. D 52, 6199 (1995).

${ }^{25}$ J. A. Schouten, Ricci Calculus (Springer, Berlin, 1954).

${ }^{26}$ L. Querella, Constrained Variations and Nonlinear Gravitational Lagrangians, Talk given at GR 15: The Fifteenth Meeting of the International Society on General Relativity and Gravitation, Poona, India, December 16-21, 1997. See also: R. Mann, Summary of Session A6, Alternative Theories of Gravity, gr-qc/9803051.

${ }^{27}$ S. Cotsakis, Mathematical Problems in Higher Order Gravity and Cosmology, Talk given at 8th Marcel Grossmann Meeting on Recent Developments in Theoretical and Experimental General Relativity, Gravitation and Relativistic Field Theories (MG 8), Jerusalem, Israel, June 22-27, 1997, gr-qc/9712046. See also: V. N. Melnikov, Multidimensional Gravity and Cosmology: Exact Solutions, gr-qc/9801037. 